\title{
Correction of Photometric Distortion of a Micro Camera-Projector System for Structured Light 3D Scanning
}

\author{
Go Gwang Park ${ }^{1}$ and Soon-Yong Park ${ }^{2,+}$
}

\begin{abstract}
This paper addresses photometric distortion problems of a compact 3D scanning sensor which is composed of a micro-size and inexpensive camera-projector system. Recently, many micro-size cameras and projectors are available. However, erroneous 3D scanning results may arise due to the poor and nonlinear photometric properties of the sensors. This paper solves two inherent photometric distortions of the sensors. First, the response functions of both the camera and projector are derived from the least squares solutions of passive and active calibration, respectively. Second, vignetting correction of the vision camera is done by using a conventional method, however the projector vignetting is corrected by using the planar homography between the image planes of the projector and camera, respectively. Experimental results show that the proposed technique enhances the linear properties of the phase patterns that are generated by the sensor.
\end{abstract}

Keywords : 3D Sensor, Photometric Distortion, Calibration, Vision Camera

\section{INTRODUCTION}

Phase-shifted fringe projection is one of the structured light 3D sensing techniques. Structured light 3D sensing is based on the triangulation between a vision camera and a pattern projection device such as laser and digital projector. For example, a digital projector projects specially coded patterns on an object of interest and a vision camera captures the image of the projected patterns. Then, to calculate the 3D coordinates of the object, the 2D coordinates of the patterns in the image planes of both the projector and the vision camera are matched[1]. To calculate the 3D coordinates of the matched pattern in the two image planes, the geometric relation between the camera and projector should be calibrated.

The geometric calibration of the 3D sensors based on the triangulation of camera-projector systems is a well-known computer vision problem and many investigations have been introduced $[2,3]$. However, only a few investigations have considered the calibration of the photometric properties of camera-projector systems. The main reason is that the most conventional structured light 3D scanning sensors employ high quality vision cameras and projectors

\footnotetext{
${ }^{1} \mathrm{AR}$-Vision Inc

${ }^{2}$ School of Computer Science and Engineering, Kyungpook National University

${ }^{+}$Corresponding author : sypark@knu.ac.kr

(Received : Jan.30, 2012, Revised : Mar. 12, 2012, Accepted : Mar. 14, 2012)
}

which have high quality photometric properties.

Nowadays, many inexpensive digital vision cameras and projectors are available. Moreover, they have also become more compact in size, and so, it is possible to develop hand-held 3D sensors. However, the compactness of the sensor inherently yields poor photometric properties such as vignetting and non-response function of camera and projector. The poor photometric properties of the sensor also yield erroneous 3D sensing results. Therefore, it is necessary to correct such properties through the photometric calibration of the sensor.

$\mathrm{W} . \mathrm{Yu}[4]$ introduces two methods of vignetting correction of a camera lens. One uses a LUT method to correct the intensity reduction in every image pixel. The other method uses a nonlinear function of lens vignetting. By inverting the nonlinear function, vignetting error is corrected. Usually, the LUT method is more commonly used than the function model. A. Litvinov et al.[5] correct vignetting and nonlinear response function problems at the same time. They derive a combination model of vignetting and nonlinear response functions. The combination model is solved by using the least square error minimization. D. Goldman[6] uses a similar method but he introduces an iterative technique for the correction of both vignetting and nonlinear response properties.

P. Debevec et al.[7] and S. Mann et al.[8] calibrate the nonlinear response functions of digital cameras to obtain 
high dynamic range images. They introduce very similar methods which use multi-exposed image to measure nonlinear image intensity with respect to the amount of light that falls on the camera sensors.

This paper addresses the photometric distortion problem of a 3D scanning sensor which consists of an inexpensive and micro-size camera-projector system. In such a cameraprojector system, photometric distortions are inherent and it could result in inaccurate $3 \mathrm{D}$ sensing. Two photometric distortions are corrected, one is a nonlinear response function and the other is vignetting. The response functions of the camera and the projector are derived from the solution of a linear equation and an illumination sensor, respectively. Vignetting of the vision camera is corrected by using a conventional technique which uses an inverting function model. To correct the projector vignetting, we use the planar homography between the image planes of the projector and the camera. Comparison of the phase image produced from the 3D sensor with and without distortion correction is shown in the experiments.

\section{DISTORTION CORRECTION OF A 3D SENSOR}

In Fig. 1, we briefly describe two photometric properties of a camera-projector system. An image $i_{x y}^{p}$ is projected from a digital projector to a planar screen. While $i_{x y}^{\prime p}$ is imaged in a micro display device such as LCD, LCOS, or DLP, a nonlinear mapping occurs from $i_{x y}^{p}$ to $i_{x y}^{\prime p}$ This is due to the response function of the micro display device. Let, $\boldsymbol{f}_{\boldsymbol{p}}$ be the response function of the projector. The distorted image $i_{x y}^{\prime p}$ then, passes through the projector lens and distorted again due to lens vignetting. As a micro-size projector is equipped with a micro-mount lens, serious vignetting distortion is inherent when an image is projected through the lens. Let $v_{x y}^{p}$ be the vignetting function of the projector. Then, the original image $i_{x y}^{p}$ becomes $i_{x y}^{\prime p}$ after it is projected to the screen.

A micro digital camera also has the same photometric distortions, nonlinear response function and vignetting. Suppose the light intensity of a scene is $i_{x y}^{c}$ The scene light is distorted by the lens vignetting, which results in the distortion from $i_{x y}^{c}$ to $i_{x y}^{\prime c}$. Then, the distorted light is distorted again in the process of converting the light intensity $i_{x y}^{\prime c}$ to an image $i_{x y}^{\prime \prime c}$. We call vignetting and nonlinear response functions of the camera as $v_{x y}^{c}$ and $\boldsymbol{f}_{c}$, respectively.

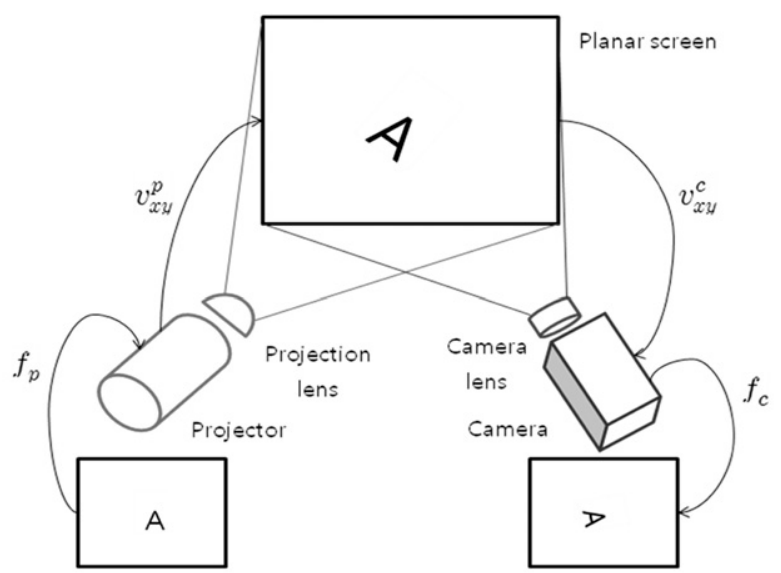

Fig. 1. Distortion functions of a camera-projector system.

\subsection{Distortion Correction of a Micro Camera}

In an ideal case, the quantity of the incoming light that falls onto the camera's imaging sensor is proportional to the exposure time $\boldsymbol{k}_{\boldsymbol{n}}$ of the camera. In other words, intensity of an image $\boldsymbol{i}_{\boldsymbol{n}}$ is $\boldsymbol{k}_{\boldsymbol{n}}$ times of a reference image $\boldsymbol{i}_{\boldsymbol{r e f}}$ such that

$$
f_{c}\left(i_{n}\right)=k_{n} f_{c}\left(i_{r e f}\right)
$$

here, $f_{c}$ is the camera's response function.

Fig. 2 shows some example images for different exposes. The left image is the reference and the other images are acquired with double-expose time.

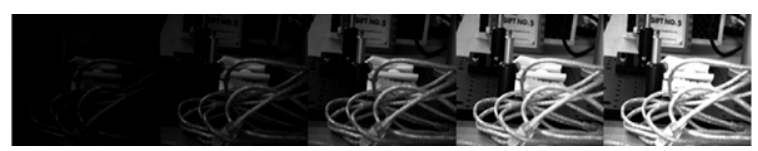

Fig. 2. Images for multiple expose times.

Taking the logarithm on both sides,

$$
F_{c}\left(i_{n}\right)-F_{c}\left(i_{r e f}\right)=K_{n}
$$

where, $\boldsymbol{K}_{\boldsymbol{n}}=\log \left(\boldsymbol{k}_{\boldsymbol{n}}\right)$. This relation suggests a way to estimate the response function from differently exposed images of the same scene as shown in Fig. 2[8].

Equation (2) gives an over-determined linear equation of the form: $\mathbf{A F}=-\mathbf{K}$, where $\mathbf{A} \in R^{L+1 \times N}, L$ is the number of pixels in $\boldsymbol{i}_{\boldsymbol{n}}$, and $N$ is the number of grayscale values (typically $N=256$ ). 256 elements of the vector $\mathbf{F}$ contain unknown transformation values of all image intensities. 
Refer to paper[8] for more details.

Once the nonlinear camera function is corrected, the vignetting of the camera lens $v_{x y}^{c}$ is corrected. Vignetting is the nonlinear reduction of brightness compared to the lens center. In an ideal case, the intensity of all the image pixels obtained from a uniform illumination should be the same. However, nonlinear intensity occurs in the image as shown in Fig. 3. The left side is an image of a reference screen $i_{x y}^{\prime c}$ The camera vignetting $v_{x y}^{c}$, can be obtained by inverting $i_{x y}^{\prime c}$ and multiplying the reference image as shown in Equation (3).
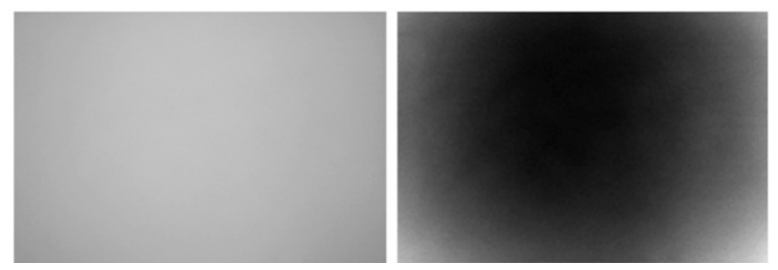

Fig. 3. Left: Image of a reference screen. Right: Amount of lens vignetting.

$$
v_{x y}^{c}=i_{0} / i_{x y}^{\prime c}
$$

To eliminate the saturation problem while correcting vignetting, we set the intensity of $\boldsymbol{i}_{0}$ to that of the center of $i_{x y}^{\prime c}$. By multiplying the vignetting function to a captured image, we obtain the original brightness of the scene such that

$$
i_{x y}^{c}=v_{x y}^{c} \times i_{x y}^{c}
$$

In our experiments, the vignetting function is obtained in advance and a LUT is used to correct the brightness of every image pixel.

\subsection{Distortion Correction of a Micro Projector}

The response function of an ideal digital projector is linear with respect to the intensity level of an image. However, most of the projectors employ nonlinear response functions to expand the dynamic range of the projectors. To correct the nonlinear function, we obtain a function $f_{p}$ and multiply its inverse to the input image before projection. Obtaining the response function is a simple task. To measure the intensity of a micro display device in the projector, an illumination sensor is used. Uniform images of intensity from 0 to 255 are projected to a white screen and their brightness is measured. To reduce the vignetting effect, brightness of the center of a projected area is measured.

Vignetting is also an inherent source of distortion in a micro projector. Correcting projector vignetting is similar to that of camera vignetting. However, projector vignetting cannot be directly measured as vignetting is only observed in a projected image, not in the display device of the projector.

Therefore, projector vignetting should be measured in a different way than that of the camera. As shown in Fig. 4, the projection area on a planar screen is captured by using the camera. The ratio of the capture area with respect to the projection area depends on the screen distance from the camera-project system. However, we set the projection area to be larger than the capture area and the capture area covers almost the total projection area.

Projector vignetting can be measured only by using the camera image. By using the image of the capture area, we correct the projector vignetting. In other words, our technique does not correct the total projection area, but instead it corrects the capture area. To implement the proposed method, a homography transform $\mathbf{H}_{\mathrm{pc}}$ from the capture area to the projection area is derived as shown in Fig. 4. The homography is then used to generate the projector image so that its projection covers only the captured area as shown in Fig. 4. Correcting projector vignetting and nonlinear response is shown in Fig. 5. A projector vignetting $v_{x y}^{p}$ is formed and it is multiplied to an input image. Then, it is transformed to be projected only in the capture area of the camera.

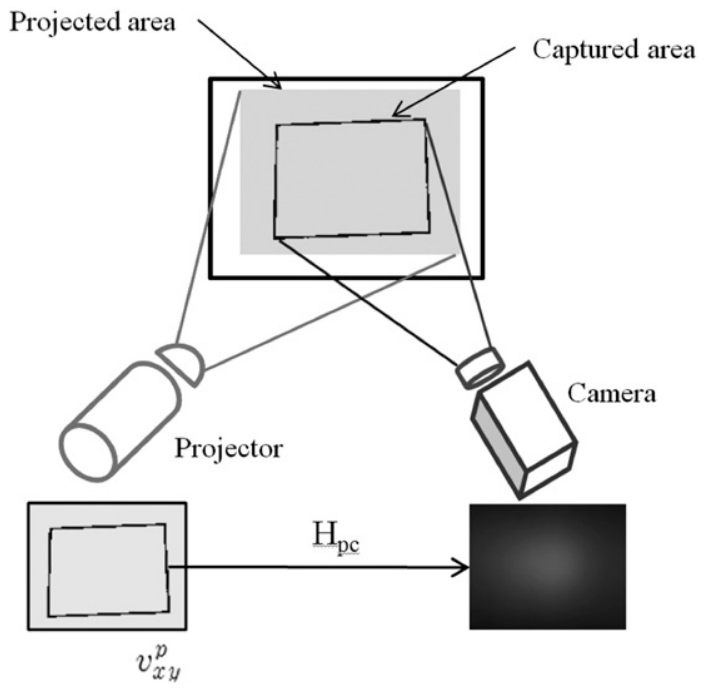

Fig. 4. Homography between projection and captured areas. 


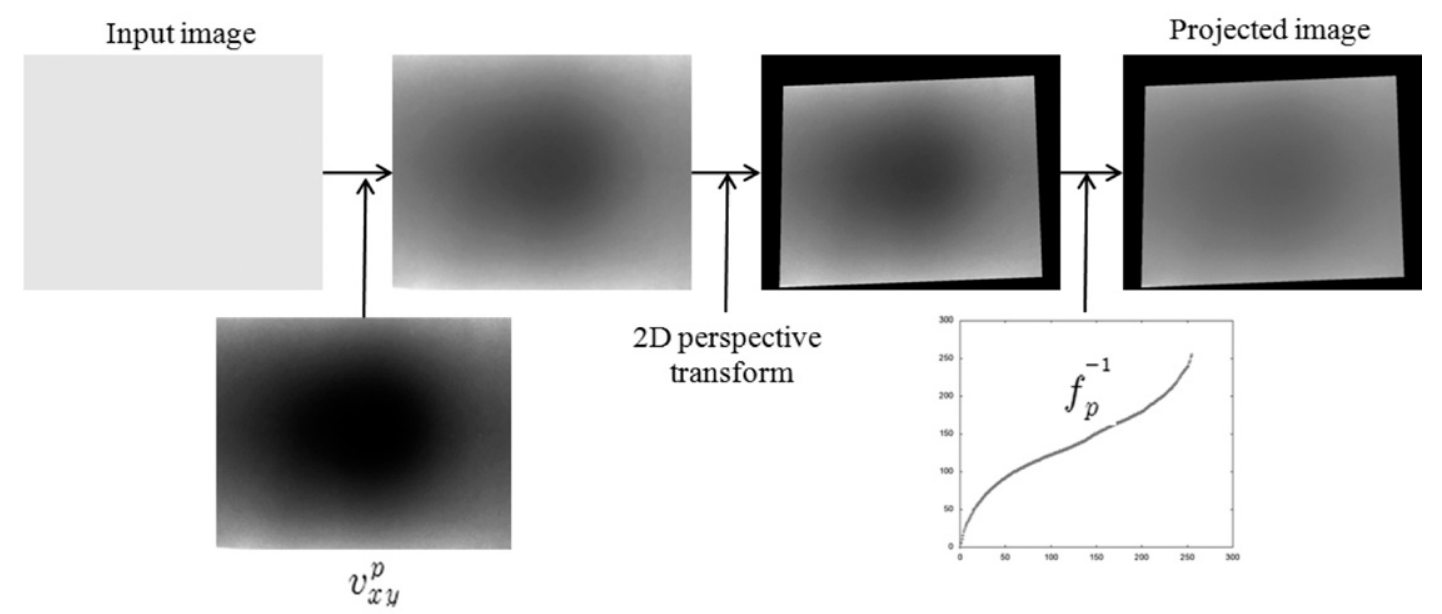

Fig. 5. Steps of distortion correction of a micro projector.

\section{EXPERIMENTAL RESULTS}

\subsection{Camera correction results}

A micro CCD camera and a micro projector are used to setup a camera-projector system as shown in Fig. 6. Both devices have the same image resolutions $1280 \times 960$. To obtain the camera response function, six differently exposed images are used to generate a comparagram as shown in Fig. 7. As we use a machine vision camera, the response function is almost linear as shown in this figure. Fig. 8 shows vignetting correction of the camera. Fig. 8(a) is the plot of a reference screen before correction and $8(\mathrm{~b})$ is after correction. As shown in the plots, intensity of all the pixels becomes almost uniform.

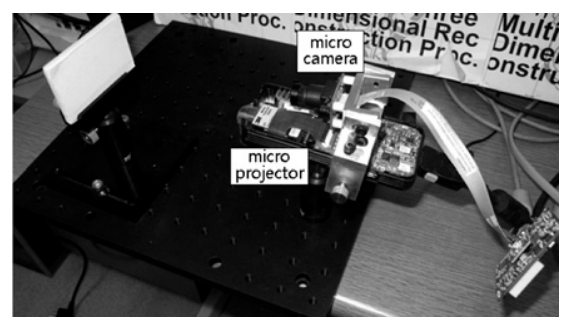

Fig. 6. Experimental setup.
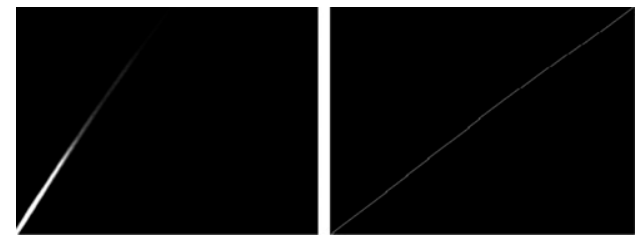

Fig. 7. Comparagram and response function of the camera

\subsection{Projector correction results}

By measuring the light intensity from the projector, we can obtain a nonlinear response curve as shown in Fig. 9. Fig. 9(a) plots the light intensity with respect to the image intensity. Fig. 9(b) plots $f_{p}^{-1}$ which is the inverse response function. The inverse function $f_{p}^{-1}$ maps the intensity levels to new corrected levels.

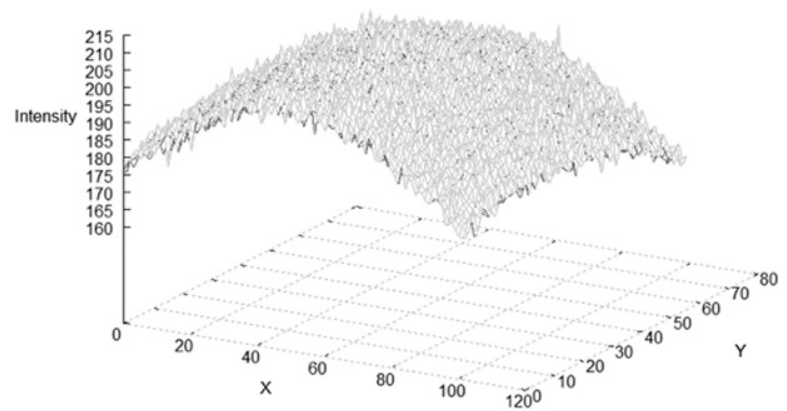

(a)

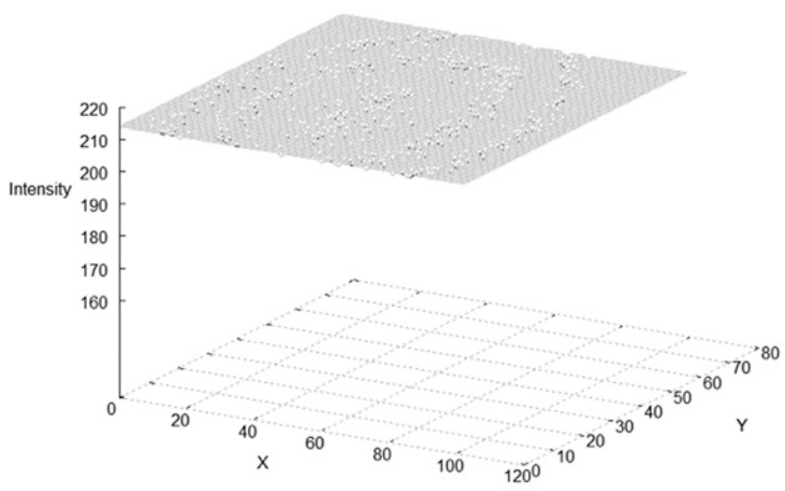

(b)

Fig. 8. Camera vignetting correction. 


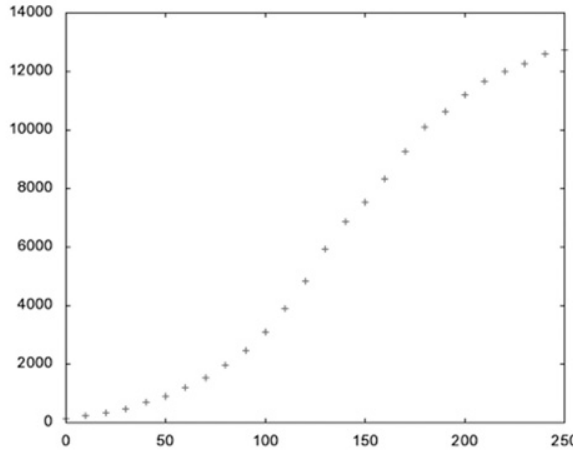

(a)

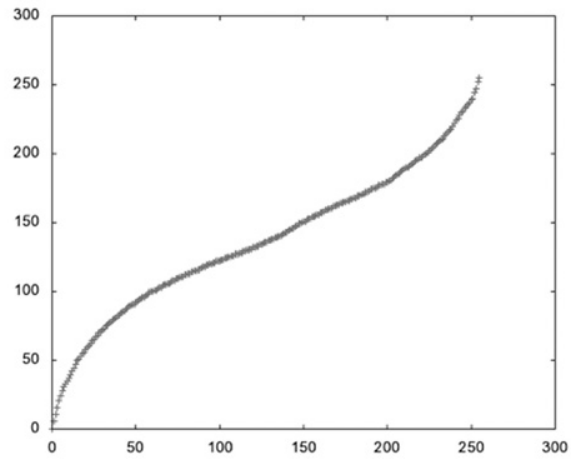

(b)

Fig. 9. Projector response function and its inverse.

Projector vignetting $v_{x y}^{p}$ is shown in Fig. 10(b). In this figure, we compare the vignetting functions of the camera and projector together. As described, to correct the camera vignetting, we multiply $v_{x y}^{c}$ to the camera images $i_{x y}^{\prime c}$. The diagonal profile of the function in Fig. 10(a) shows that the intensity of the boundary pixels is amplified with respect to the reference level (1.0).
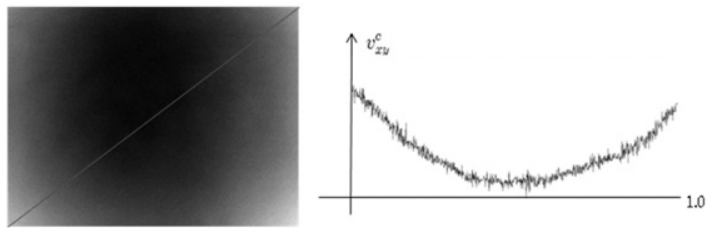

(a)
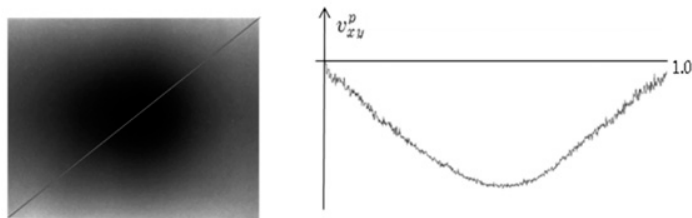

(b)

Fig. 10. Comparison of camera and projector vignetting.

However, vignetting correction of projector is different. As shown in the diagonal profile of $v_{x y}^{p}$ in Fig. 10(b), the intensity of the center pixels is reduced. As the intensity of the reference image is the maximum intensity level of the projector, we cannot amplify the boundary pixels. Instead, we reduce the intensity of the center pixels to uniformly display a reference image. Fig. 11 shows vignetting correction results. As seen from the plots, the distorted intensity levels are corrected in the result image. In the plots, the intensity of the center area is reduced.

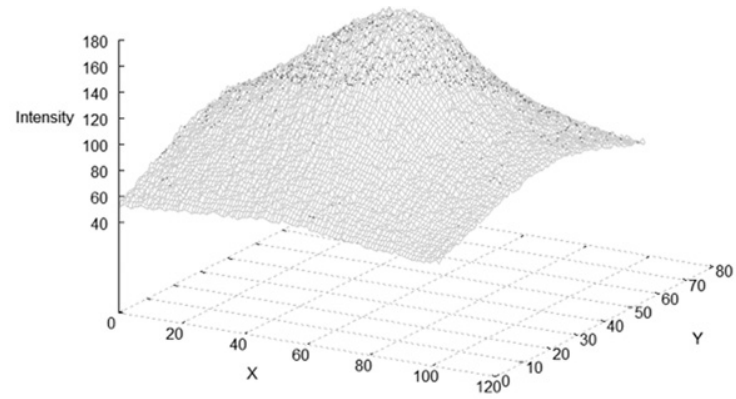

(a)

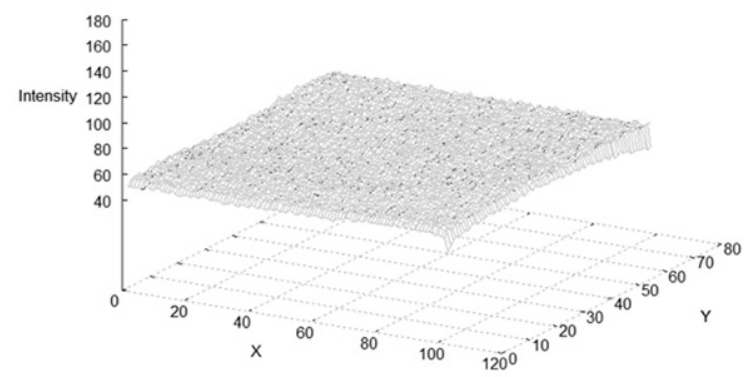

(b)

Fig. 11. Projector vignetting correction.

\subsection{Comparison of the phase shifted images}

An important application of our distortion correction method is structured light 3D scanning. We are especially developing a 3D scanning system which is based on the phase shifted fringe projection[9]. Three phase images are generated and projected sequentially to get phase images which are shown in Fig. 12. The 2D plots in the figure show intensity values along the horizontal line in each phase image. Without correction, the phase values are not linear. After correction, phase values of the same horizontal lines become almost linear. By using the proposed correction technique, accurate phase images can be generated and they will provide accurate $3 \mathrm{D}$ scanning results. 


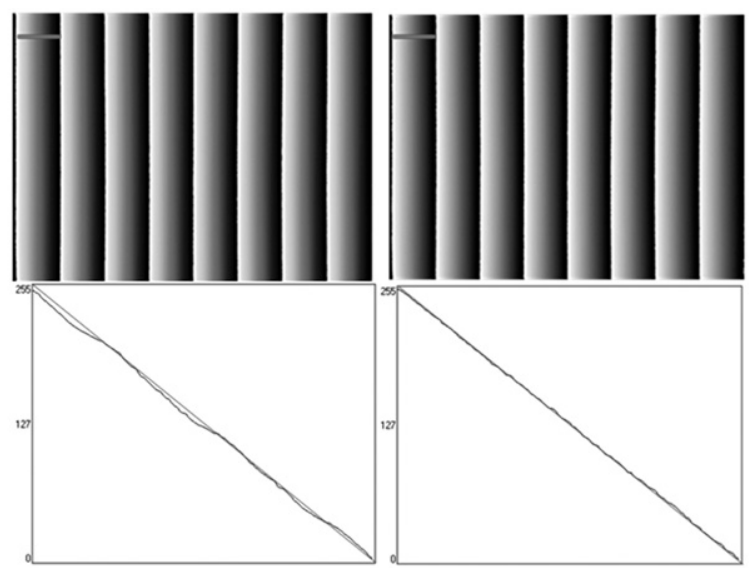

Fig. 12. Comparison of phase images. Left: Before correction. Right: After correction.

\section{CONCLUSION}

In order to develop an accurate 3D scanning sensor, photometric distortions of a camera-projector system were corrected. Nonlinear response functions and vignetting of both camera and projector are calibrated and corrected. Passive and active calibration techniques are used to derive the response functions. Homography between projection and capture areas is derived to correct the projector vignetting from the view of the camera. Experimental results show the corrections of the photometric distortions of the system and enhanced linearity in a phase map which is produced by a $3 \mathrm{D}$ sensor.

\section{ACKNOWLEDGMENT}

This work was supported by the Korea Research Foundation Grant funded by the Korean Government(KRF -2008-313-D00959)

\section{REFERENCES}

[1] J. Pages, J. Salvi, R. Garcia, and C. Matabosch, "Overview of coded light projection techniques for automatic 3D profiling”, IEEE International Conference on Robotics and Automation, 2003.

[2] R. Sukthankar, R. G. Stockton, and M. D. Mullin, "Smarter presentations: Exploiting homography in camera-projector systems", Proceedings of International Conference on Computer Vision, vol. 1, pp. 247-253, 2001.

[3] R. Raskar and P. Beardsley, "A self correcting projector", IEEE Computer Vision and Pattern Recognition, pp. 626-631, 2001.

[4] W. Yu, "Practical anti-vignetting methods for digital cameras", IEEE Trans. on Consumer Electronics, vol. 50, no. 4, pp. 975-983, 2004.

[5] A. Litvinov and Y. Y. Schechner, "Addressing radiometric nonidealities: A unified framework", Proc. IEEE Conf. Computer Vision and Pattern Recognition, vol. II, pp. 52-59, 2005.

[6] D. B Goldman and J. H. Chen, "Vignette and exposure calibration and compensation", Proc. IEEE Int. Conf. on Computer Vision, pp. 899-906, 2005.

[7] P. E. Debevec and J. Malik, "Recovering high dynamic range radiance maps from photographs", SIGGRAPH, 1997.

[8] S. Mann and R. Mann, "Quantigraphic imaging: Estimating the camera response and exposures from differently exposed images", Proc. IEEE Conf. Computer Vision and Pattern Recognition, pp. 842849, 2001.

[9] P. S. Huang and S. Zhang, "Fast three-step phaseshifting algorithm", Appl. Opt. vol. 45, Issue 21, pp. 5086-5091, 2006. 
Go Gwang Park received his B.S and M.S. degrees in the School of Electrical Engineering and Computer Science from Kyungpook National University, Daegu, Korea, in 2009 and 2011, respectively. He is currently a research staff at AR-Vision Inc., Daejeon, Korea. His research interests are in 3D Sensing and Modeling.

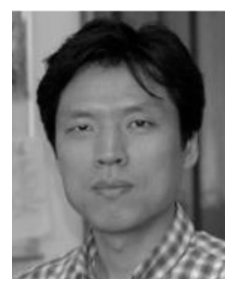

Soon-Yong Park received his B.S. and M.S. degrees in Electronics Engineering from Kyungpook National University, Daegu, Korea, in 1991 and 1999, and his Ph.D. degree in Electrical and Computer Engineering from State University of New York at Stony Brook in 2003. From 1993 to 1999, he was a senior research staff at KAERI, Korea. He is currently an associate professor in the School of Computer Science and Engineering, Kyungpook National University. His research interests include 3D Sensing and Modeling, Multi-view 3D Data Processing. 